\title{
Armando Yarlequé Chocas
}

\section{Leonardo Romero}

Editor Jefe, Instituto de Investigación de Ciencias Biológicas Antonio Raimondi, Facultad de Ciencias Biológicas, Universidad Nacional Mayor de San Marcos. Apartado 11-0058, Lima 11, Perú. Email: Iromeroc@unmsm.edu.pe

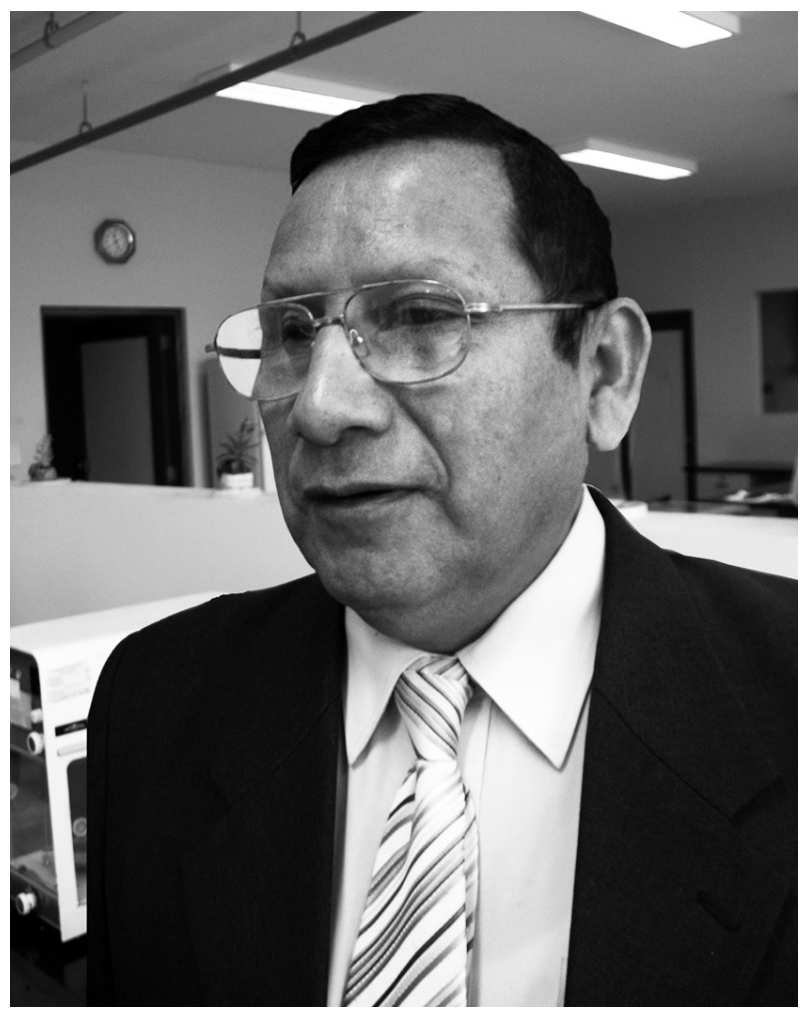

Armando Yarlequé es un científico sanmarquino que resalta por sus aportes, logros y capacidades, los que adquieren especial valor porque son una respuesta exitosa a las condiciones adversas que una enfermedad ocular (retinosis) le ha impuesto, y que nos muestran un ejemplo de persona, investigador, profesor, académico y ser humano decidido a dejar huella en esta vida. Fue el primer Editor Jefe de la Revista Peruana de Biología después que la Asociación de Biólogos de San Marcos cediera los derechos a la Facultad de Ciencias Biológicas y en las siguientes palabras rendiremos homenaje a su trayectoria.

Armando Yarlequé Chocas nació el 5de mayo de 1948, su vida académica y profesional se inició en 1972 cuando obtuvo el grado de bachiller en Ciencias Biológicas y su título profesional de Biólogo. En este mismo año ingreso a la docencia. Su interés por la investigación en bioquímica y biología molecular adquirió cuerpo cuando en 1976 asumió la Jefatura del Laboratorio de Biología Molecular, de la Facultad de Ciencias Biológicas de la Universidad Nacional Mayor de San Marcos, laboratorio que ha conducido hasta el presente convirtiéndolo en uno de los más productivos en publicaciones científicas y tesis, tanto de pregrado como de postgrado. En el año 1985 fue becario del Consejo Británico en el Queen Elizabeth College, Universidad de Londres. En el año 1987 obtuvo el grado de doctor en Ciencias Biológica con la tesis "Enzima similar a trombina del veneno de la serpiente peruana Lachesis muta: Aislamiento, Caracterización, Bioquímica y Acción Biológica”.

Armando Yarlequé también ha manifestado interés en la organización y la política de investigación, como parte de un compromiso de la Universidad con la sociedad peruana. En 1986 fue elegido Director del Instituto de Ciencias Biológicas Antonio Raimondi (ICBAR) y desempeñó el cargo hasta el año 2001. En este periodo se dieron inicio a las Reuniones Científicas del Instituto que recientemente en el 2011 llegaron a la XX edición. Las Reuniones Científicas se han convertido en un evento académico de mucha importancia en el Perú y que suelen congregar a cientos de investigadores para intercambiar ideas e información.

Desde el año 2005 ha ocupado diversos cargos en la dirección de nuestra Universidad, la mayoria de ellos ligado a la investigación, fue Asesor de la Oficina General de Cooperación y Relaciones Interinstitucionales, Asesor de la Oficina General de Admisión, Jefe de la Oficina de Coordinación de Servicios de Investigación e Innovación, y recientemente entre el año 2009 y 2011 fue Presidente del Consejo Superior de Investigación.

Armando Yarlequé fue Editor Jefe de la Revista Peruana de Biología en el periodo de 1998 al 2001. En ese periodo también era Director del Instituto de Ciencias Biológicas y a él le toco 
asegurar los principios y elaborar la política editorial de la revista, aspecto que casi no ha cambiado hasta el presente.

Como una actividad de extensión, en 1993 implemento el Serpentario Oswaldo Meneses que tiene sede en el Museo de Historia Natural y lo dirige hasta la fecha.

Ha participado en la coordinación y ejecución de diversos convenios de nuestra Universidad como los realizados con: el Queen Elizabeth College (1984 - 1990), la Academia de Ciencias de la Ex Unión Soviética (1986 - 1992), el Research Institute of Chemistry - University of Karachi, Pakistan (1990 - 1994), el Instituto de Investigaciones Biológicas Clemente Estable de Uruguay (1992 - 2006), la Fundação Ezequiel Dias (Funed-Brasil) (2000 - hasta la fecha), el Centro de Red Iberoamericana sobre venenos y antivenenos ofídicos, San José de Costa Rica, CYTED (2005 - 2010), las Universities of Oxford and Liverpool (2000 - 2005), el Instituto nacional de Salud $(2004-2011)$

Su actividad como investigador y docente ha sido reconocida, tanto fuero como dentro de la Universidad; le han sido otorgados el Primer Premio en el área de Ciencias afines a la Salud de la Fundación Hipólito Unanue (1984); el Premio en el Área de Ciencias Biológicas otorgado por la Academia de Ciencias para el Tercer Mundo - TWAS (1987); la Distinción otorgada por la Municipalidad de Lurigancho - Chosica entre los 100 personajes ilustres, con motivo del centenario del Distrito (1995); el Primer Premio en el área de Ciencias afines a la Salud otorgado por la Fundación Hipólito Unanue (1996); el Premio como Científico más destacado entre 1998 - 1999 en Ciencias Biológicas y en el área de Ciencias Básicas otorgado por la UNMSM (2000); y Reconocimiento al merito científico otorgado por el Colegio de Biólogos del Perú lo recibió en dos oportunidades en el año 2001 y en 2009.

Es miembro de la Sociedad Química del Perú, la Sociedad Internacional de Toxinología (IST), la Sociedad Internacional de Ecología Química (ISCE), la Red Latinoamericana de Productos Naturales (LANBIO), el Colegio de Biólogos del Perú, la Sociedad Peruana de Inmunología y la Products Research Networks in Africa, Asia and South America (AFASSA) .

Armando Yarlequé es una persona muy disciplinada, de puntualidad y tiempos exactos, decidido a realizar y cumplir objetivos. Pero a la vez es capaz de dar un tiempo para orientar a sus alumnos, escuchar a los amigos y discutir sobre ideas. Gusta de leer y dedicarle mucho tiempo a buscar información, preparar sus clases y organizar sus cursos; en esto último es un implacable organizador, perseverante en los detalles y un acicate con sus asistentes. En estos momentos en que los medios de información solo dan cuenta de las enfermedades de nuestra sociedad y sistemas quebrados por la falta de principios y la inversión de la moral, seres como Armando Yarlequé nos recuerdan el compromiso de los investigadores, los académicos y los sanmarquinos con la sociedad; nos marcan un ritmo, porque nos falta mucho por lograr; nos señalan problemas, de los muchos que nos faltan por resolver; nos muestran lo que hemos hecho porque sólo continuando la obra podremos avanzar y respetarnos a nosotros mismos como comunidad. Por eso, este homenaje es además como una clase a nuestros alumnos, una clase sin horario ni aula, pero que ellos podrán entender y sentir en cada momento y lugar.

\section{Publicaciones de Armando Yarlequé en libros y revistas especializadas}

1. Yarlequé A. \& S. Campos. 1973. Actividad de una Fosfodiesterasa en el veneno de la serpiente Lachesis muta. Boletín de La Sociedad Química del Perú. XXXIX(3):141-147.

2. Campos S. \& A. Yarlequé 1974. 5'nucleotidasa en el veneno de la serpiente Lachesis muta. Boletín De La Sociedad Química Del Perú. XL(3): 202-212.

3. Morante Y. \& Yarlequé A. 1980. Proteasas del veneno de serpientes influencia de algunos agentes químicos en la actividad proteolítica del veneno de Lachesis muta "Shushupe". Acta Científica Venezolana. 31: 148-153.

4. Heredia V., Campos S. \& Yarlequé A. 1982. Actividad de una 5 'nucleotidasa en el veneno de Bothrops atrox (L) "Jergón". Acta Cientifica Venezolana. 33: 333-347.

5. Yarlequé A., Escobar E. \& Campos S. 1983. Exonucleasas y otras actividades nucleolíticas en los venenos de Lachesis muta y Bothrops atrox. Acta Cientifica Venezolana. 34: 336-340.

6. Cruz L. \& Yarlequé A. 1984. Hemólisis de eritrocitos humanos por acción del veneno de Lachesis muta y Bothrops atrox. Boletin De La Sociedad Quimica Del Peru. L(1): 41-48.

7. Zavaleta A., O. Castro De La Mata, M. Salas, R. Castro De La Mata \& A. Yarlequé. 1984. Loxocelismo experimental: Aspectos farmacológicos y anatomopatológicos. Diagnostico. 14(6): 163-173.

8. Loayza S., Y. Morante, S. Campos \& A. Yarlequé. 1985. Enzimas proteolíticas en el veneno de las serpientes peruanas Lachesis muta y Bothrops atrox. Boletin De La Sociedad Quimica Del Peru. LII (3): 151-163.

9. Yarlequé A., V. Heredia, E. Arvaiza, S. Campos \& A. Zavaleta. 1985. Contenido proteico y actividades enzimáticas presentes en el veneno de la araña casera Loxoceles laeta (II parte). Diagnostico. 15(1): 5-9.

10. Herrera E., A. Yarlequé, C. Campos \& A. Zavaleta. 1986. Efecto de la radiación gamma sobre la actividad biológica y propiedades enzimáticas de los venenos de las serpientes Lachesis muta y Bothrops atrox. Informe Nuclear. 3(1): 1-14.

11. Yarlequé A., V. Heredia, E. Arvaiza \& A. Zavaleta.1986. Estudios electroforéticos y Acción procoagulante del veneno de Loxoceles laeta. Diagnostico. 17(2): 39-45.

12. Gomez De La Torre G., A. Zavaleta, R. Castro De La Mata, M. Arana \& A. Yarlequé. 1986. El conejo: Un modelo experimental de Loxocelismo cutáneo y viscerohemolítico. Diagnostico. 18(3): 65-73.

13. Barboni E., D.M. Kemeni, S. Campos \& C.A. Vernon. 1987. The purification of acid phosphatase from Honey Bee venom (Apis mellifera). Toxicon. 25(10): 1097-1103.

14. Escobar E., O. Malaga \& A. Yarlequé. 1987. Purificación y propiedades de una exonucleasa del veneno de la serpiente Lachesis muta. Boletín de la Sociedad Química Del Perú. LIII(1): 1-14.

15. Paredes R., A. Zavaleta, M. Mendoza, M. Salas, A. Yuen, A. Yarlequé \& E. Rosas. 1987. Loxocelismo experimental: Efectos sobre el sistema de coagulación sanguínea. Diagnostico. 20(2):50-53.

16. Yarlequé A. 1987. Enzima similar a trombina del veneno de la serpiente peruana Lachesis muta: Aislamiento, Caracterización, Bioquímica y Acción Biológica. Tesis Doctoral. Facultad de Ciencias Biológicas. Universidad Nacional Mayor de San Marcos.

17. Arbaiza E., C. Giusti, A. Yarlequé, N. Martinez, A.B. Dudelina, A.V. Kisher \& E.V. Grishin 1988. Fraccionamiento y caracterización parcial del veneno de la Hormiga Paraponera clavata "Isula". Boletín de la Sociedad Química del Perú. LIV(4): 229-243. 
18. Campos S., E. Escobar, F. Lazo, A. Yarlequé, N.A. Marsh, P.M. Peyser, B.C. Whaler, L.J. Creighton, P.J. Gaffney. 1988. Partial separation of a thrombin-like enzyme from the venom of the Peruvian bushmaster snake, Lachesis muta muta. In: Hemostasis and Animal Venoms. Pirkle H, Markland FS, Jr, (eds). Marcel Dekker, New York. 107-15.

19. Heredia V., E. Arbaiza, J. Venegas, A. Yarlequé \& A. Zavaleta. 1989. Aportes al estudio de las acciones proteolíticas procoagulantes y caracterizaciones electroforéticas de las proteínas de 2 extractos tóxicos de veneno de Loxoceles laeta. Bol. Chil. Parasitol. 44: 8-16.

20. Yarlequé A., S. Campos, E. Escobar, F. Lazo, N.S. Sanchez, N.N. Marsh, P. Butterworth \& R. Price. 1989. Isolation and characterization of a Fibrinogen-Clotting enzyme from venom of the snake Lachesis muta muta (Peruvian Bushmaster). Toxicon. 27(11):1189-1197.

21. Rodriguez E. \& A. Yarlequé. 1991. Aislamiento y algunas propiedades de la proteinasa I del veneno de la serpiente peruana Lachesis muta. Acta Cientifica Venezolana. 42: 219-225.

22. Escobar E., E. Rodrigues, A. Yarlequé. 1992. Isolation and partial characterization of a fibrinogenase from the venom of the Peruvian Bushmaster Lachesis muta. In: Gopalakrishnakone P., Tan Choon Kin. Recent Advances in Toxinology Research., Singapore, National University of Singapore: 421-430.

23. Roncalla R. \& Yarlequé A. 1995. Efecto del ácido glutámico sobre algunas enzimas de los venenos de serpientes. Boletín de la Sociedad Química del Perú. LXI(1): 38-47.

24. Cardenas J., C. Pantigoso, O. Malaga \& A. Yarlequé. 1995. Contenido proteico y algunas propiedades enzimáticas en tres venenos de serpientes mantenidas en cautiverio. Boletín de la Sociedad Química del Perú. LXI(3):151-163.

25. Pantigoso C., E. Escobar, O. Malaga \& A. Yarlequé. 1996. Aislamiento y algunas propiedades de la atroxina una proteinasa del venenos de la serpiente peruana Bothrops atrox “Jergón”. Acta Cientifica Venezolana. 47: 67-73.

26. Lazo F., E. Rodriguez \& A. Yarlequé. 1998. Evaluación comparativa de 2 métodos para determinar la actividad de Fosfolipasa A en venenos de serpientes. Revista Peruana de Biología. 5(2): 98-102.

27. Paredes C., I. Garate \& A. Yarlequé. 1999. Actividad in vitro de los venenos de la serpiente Lachesis muta y Bothrops atrox sobre la viabilidad y desarrollo embrionario de los huevos de Ascaris suum. Revista Peruana de Biología 6(1): 85-93.

28. Solis Ch., Escobar E., Yarlequé A. \& Gutierrez S. 1999. Purificación y caracterización de la L-aminoácido oxidasa del veneno de la serpiente Bothrops brazili "Jergón Shushupe". Revista Peruana De Biologia. 6(1): 75-84.

29. Zevallos J., E. Escobar, O. Malaga \& A. Yarlequé. 1999. Aislamiento y algunas propiedades de una fosfolipasa del veneno de la serpiente Bothrops brazili. Boletín de la Sociedad Química del Perú. LXV(1): 10-20.

30. Azañero A., E. Escobar \& A. Yarlequé. 2000. Purificación de una enzima proteolítica del veneno de Bothrops brazili y estudio de su actividad sobre fibrinógeno. Revista Peruana De Biologia. 7(1): 55-66.

31. Malaga O., C. Pantigoso, Y. Morante, V. Heredia, J. Cardenas \& A. Yarlequé. 2000. Variaciones en la composición proteica actividades enzimáticas y biológicas del veneno de la serpiente Bothrops atrox (Viperidae) en relación con la edad. Revista Peruana de Biología. 7(2): 161-170.

32. Remuzgo C., M. Alvarez, F. Lazo \& A. Yarlequé. 2000. Caracterización parcial del veneno de la serpiente cascabel peruana Crotalus durissus terrificus. Revista Peruana de Biología. 7(1): 67-73.
33. Yarlequé A. 2000. Las serpientes Peruanas y sus Venenos. Fondo Editorial de la UNMSM. Lima.

34. Pantigoso C., E. Escobar \& A. Yarlequé. 2001. Aislamiento y caracterización de una miotoxina del veneno de la serpiente Bothrops brazili Hoge 1953 (Ophidia: Viperidae). Revista Peruana de Biología. 8(2): 136-148.

35. Remuzgo C., Alvarez M. P., Rodríguez E., Lazo F. \& Yarlequé A. 2001. Micrurus spixii (peruvian coral snake) venompreliminary biochemical and enzymatic characterization. The Journal ofVenomous Animals and Toxins. 8(2): 51-66.

36. Yarlequé A., A. Alvarez, C. Remuzgo, F. Lazo \& E. Rodríguez. 2002. Aislamiento y caracterización parcial de dos proteínas presentes en el veneno de la "Serpiente coral" peruana Micrurus spixii. Boletín de la Sociedad Químicas del Perú. 68(2): 74-83.

37. Isla M., O. Malaga \& A. Yarlequé. 2003. Caracteristicas bioquímicas y acción biológica de una hemorragina del veneno de Bothrops brazili. Anales de la Facultad de Medicina. 64(3): 159-166.

38. Magalhaes A., R. Ferreira., M. Richardson, S. Gontijo, A. Yarlequé, H. Magalhaes, Bloch. \& E. Sánchez. 2003. Coagulant thrombin-like enzymes form the venoms of Brazilian and Peruvian bushmaster (Lachesis muta muta) snakes. Comparative Biochemestry and Physiology. Part B. 136: 255-266.

39. Pantigoso C., E. Escobar \& A. Yarlequé 2003. Acción de la miotoxina del veneno Bothrops brazili Hoge 1953 (Ophidia: Viperidae) Revista Peruana de Biología 9(2): 74-83.

40. Huatuco S., E. Escobar \& A. Yarlequé. 2004. Aislamiento y caracterización parcial de una miotoxina del veneno de la serpiente Bothrops atrox (Ophidia: Viperidae). Revista Peruana de Biología 11(1): 79 - 86.

41. Laing G.D. Yarlequé A. Marcelo A. Rodríguez E. Warrell D.A. \& Theakston R.D.G. 2004. Preclinical testing of three South American antivenoms against the venoms of five medically -important Peruvian snake venoms. Toxicon. 44:103-106.

42. Lerma L., G. Sandoval, R. Inga, M. Muñoz, E. Cavero \& A. Yarlequé. 2004. Análisis cromatográfico del contenido enzimático del veneno de tres serpientes peruanas. Arnaldoa 11(2): $105-116$.

43. Roncalla R. E. Rodriguez , L. Lerma \& A. Yarlequé. 2005. Purificación parcial y características bioquímicas de acetilcolinesterasa presente en los venenos de las serpientes Micrurus spixxii y Hemachatus haemachatus. Revista de la Sociedad Química del Perú. 74(4):155-265.

44. Cisneros Y., F. Lazo, S. Gutierrez \& A. Yarlequé. 2006. Características Bioquímicas de una proteína Antibacteriana aislada del veneno de Lachesis muta "Shushupe". Revista de la Sociedad Química del Perú. 72(34):187-196.

45. Hermogenes A., M. Richardson, A. Magalhaes, A. Yarlequé, E. Rodriguéz, \& E. Sánchez. 2006. Interaction of plasminogen activator proteinase LV-PA with human $\alpha 2$ macroglobulin. Toxicon 47: 490-494.

46. Mejía J., R. Inga, F. Lazo, E. Rodriguéz, A. Yarlqué \& A. Zavaleta. 2006. Purificación y propiedades bioquímicas de una Fosfolipasa A del veneno de la serpiente Lachesis muta "Shushupe". Revista de la Sociedad Química del Perú. 72(2):86-95.

47. Sandoval G., L. Lerma, E. Rodriguéz, A. Yarlequé, Y. Espinoza, H. Solis \& W. Roldan. 2006. Purificación de anticuerpos policlonales contra el veneno de la serpiente peruana Bothrops atrox (“Jergón”) por cromatografía de afinidad. Revista de la Sociedad Química del Perú. 72(3):140-149.

48. Garate I., A. Naupay, B. Suyo, H. Colquichagua, E. Rodriguez \& A. Yarlequé. 2007. Identificación de Porocephalus stilessi (Pentastomida) en la serpiente peruana Lachesis muta. Rev. Inv. Vet. Peru. 18(2): 89-93. 
49. Hurtado L., L. Lerma, E. Rodríguez \& A. Yarlequé. 2007 Aislamiento y algunas propiedades bioquímicas de una Hialuronato glicanohidrolasa del veneno de la serpiente Lachesis muta Shushupe. Revista de la Sociedad Química del Perú. 73(4):226-234.

50. Lazo F., O. Malaga, A. Yarlequé, \& R. Severino. 2007. Algunas propiedades bioquímicas de una L-aminoácido oxidasa aislada del veneno de la serpiente Bothrops atrox. Revista de la Sociedad Química del Perú. 73(3):131-141.

51. Lazo F., O. Malaga, A. Yarlequé, R. Severino \& S. Gutierrez. 2007. Actividad antimicrobiana de una flavo proteína aislada del veneno de la serpiente peruana Bothrops atrox (Jergón). Revista de la Sociedad Química del Perú. 73(4):197-207.

52. Monteghirfo M. \& A. Yarlequé-Chocas. 2007. Caracterización de las proteínas totales de tres ecotipos de maca (Lepidium peruvianum G. Chacón), mediante electroforesis unidimensional y bidimensional. An. Fac. med. 68(4):301-306.

53. Ponce-Soto L.A., V.L. Bonfim, J.C. Novello, R. Navarro Oviedo, A. Yarlequé Chocas, \& S. Marangoni. 2007. Isolation and Characterization of a Serine Protease, Ba III-4, from Peruvian Bothrops atrox venom. The Protein Journal 26: 387-394.

54. Garcia P., A. Yarlequé, C. Bonilla, S. Pessah, D. Vivas, G. Sandoval, \& F. Lazo. 2008. Características bioquímicas y evaluación preclínica de un antiveneno botrópico liofilizado contra el veneno de la serpientes Bothrops atrox. Rev. Peru Med. Exp. Salud Pública 25(4): 386-90.

55. Mendoza J., F. Lazo, L. Yarlequé, N. Ruiz, A. Yarlequé, S. Pessah, V. Flores \& C. Bonilla. 2008. Efecto del antiveneno botrópico sobre las actividades de Fosfolipasa A2 L-aminoácido oxidasa y hialuronidasa de los venenos de serpientes peruanas. Rev. Peru Med. Exp. Salud Pública 25(2): 174-78.

56. Yarlequé A, D. Vivas, R. Inga, E. Rodriguez, G. Sandoval, S. Pessah \& C. Bonilla-Ferreyra. 2008. Acción del antiveneno botrópico polivalente sobre las actividades proteolíticas presentes en los venenos de serpientes peruanas. Rev. Peru Med. Exp. Salud Pública 25(2): 169-73.

57. Mendoza J., D. Vivas, R. Inga, E. Arbaiza, E. Rodriguez \& A. Yarlequé. 2009. Patrones electroforéticos de los venenos de serpientes peruanas de los géneros Bothrops y Lachesis. Revista de la Sociedad Química del Perú. 75(2):235-42.
58. Inga R., D. Vivas, P. Palermo, J. Mendoza, F. Lazo \& Yarlequé A. 2010. Caracterización biológica y acción de inhibidores de una fosfolipasa A2 del veneno de Lachesis muta. Revista Peruana de Biología. 17(1): 123-128.

59. Jimenez K. L., A.I. Zavaleta, V. Izaguirre, A. Yarlequé \& R. Inga. 2010. Clonaje y caracterización molecular in silico de un transcripto de fosfolipasa A2 aislado del veneno de la serpiente peruana Lachesis muta. Revista Peruana De Medicina Experimental En Salud Pública. 27(4): 532-539.

60. Rodriguez E., G. Cahuana, G. Sandoval, M. Yarlequé \& A. Yarlequé. 2010. Evaluación preliminar de la actividad coagulante del veneno de la serpiente peruana "loro machaco". 76(2):131-137.

61. Ruiz N., C. Solis, G.A. Sandoval, F. Lazo, E. Rodriguez \& A.Yarlequé 2010. Algunas propiedades cinéticas de una L-aminoácido oxidasa purificada del veneno de la serpiente peruana Bothrops atrox “Jergón". Revista de la Sociedad Química del Perú. 76(3): 218-226.

62. Sanchez E. F., F.S. Schneider, A. Yarlequé, M.H. Borges, M. Richardson, S.G. Figueiredo, K.S. Evangelista, \& J.A. Eble. 2010. The novel metalloproteinase atroxlysin-I from Peruvian Bothrops atrox (Jergón) snake venom acts both on blood vessel ECM and platelets. Archives of Biochemistry and Biophysics 496 (1): 9-20.

63. Sandoval G.A., F. Lazo, E. Rodriguez, A. Yarlequé \& R. B. Zingali. 2010. Identificación molecular y actividad sobre sustratos cromogénicos de la venombina A del veneno de la serpiente peruana Bothrops atrox. Revista Peruana de Biología. 17(3): 365-370.

64. Sandoval G.A., N. Ruiz, F. Lazo, E. Rodriguez, A. Yarlequé \& R.B. Zingali. 2010. Aislamiento y caracterización parcial de una enzima similar atrombina del veneno de la serpiente peruana bothrops atrox "jergón". Revista de la Sociedad Química del Perú.76(2):156-164.

65. Segura A., M.C. Castillo, V. Núñez, A. Yarlequé, L.R.C. Gonçalves, M. Villalta, C. Bonilla, et al. 2010. Preclinical assessment of the neutralizing capacity of antivenoms produced in six Latin American countries against medicallyrelevant Bothrops snake venoms. Toxicon 56(6): 980-989.

66. Vivas Ruiz D., R. Inga Arellano, J. Mendoza Fernandez, F. Lazo Manrique \& A. Yarlequé. 2010. Barnetobina: Un nuevo principio coagulante purificado del veneno de la serpiente peruana Bothrops barnetti. Revista de la Sociedad Química del Perú. 76(3): 261-270. 